\title{
A Model of Wireless Sensor Networks using Opportunistic Routing in Logistic Harbor Scenarios
}

Vo Que Son, Bernd-Ludwig Wenning, Andreas Timm-Giel, Carmelita Görg

Communication Networks (ComNets)

University of Bremen, Bremen, Germany

\{son,wenn, atg, cg $\} @$ comnets.uni-bremen.de

\begin{abstract}
Collection of information for management in logistics is of great importance today. Many applications in logistics are currently starting to use modern technologies such as RFID. However, these technologies have not provided enough information for management such as the real-time condition of goods or online item tracking during transport. In this paper, a model for Wireless Sensor Networks (WSNs) in logistics is proposed under the consideration of mobility and multiple WSN domains. With the use of WSNs, relevant information can be monitored down to the level of individual logistic items. For the communication reliability of sensor networks, a high packet reception ratio (PRR) is desired. To achieve this high PRR, many techniques are used in our model to conserve energy and to improve the reliability. The results show that a PRR of $90 \%$ can be reached in our simulation scenarios using the proposed model. Moreover, a logistic scenario in a harbor is examined as an example to investigate the capability to connect automatically in multiple sensor networks.
\end{abstract}

Keywords: - Wireless Sensor Networks, opportunistic routing, logistics.

\section{Introduction}

With their rapid development, Wireless Sensor Networks (WSNs) have gone beyond the scope of monitoring the environment [3], [4], [5]. A WSN is a wireless network consisting of spatially distributed autonomous devices using sensors to 
cooperatively monitor physical or environmental conditions, such as temperature, sound, vibration, pressure, at different locations. These sensor nodes can form a self-organizing network which fits well in mobile environments. Having some advantages (e.g. low power consumption and multi-hop routing), WSNs allow telemetry, control and management applications which can be widely used in logistics, especially in autonomous logistic systems. Multi-hop routing protocols for sensor networks can in most cases be classified into collection and dissemination protocols [10]. In this paper we only discuss the collection protocols.

Directed Diffusion, the earliest WSN routing protocol, sets up a collection tree based on data specific node requests [11]. Early experiments led many deployments to move towards a simpler and less general approach [10]. Second generation protocols such as MintRoute [14] use periodic broadcasts to estimate the transmissions per delivery on a link. MultiHopLQI is a third generation protocol which adds physical layer signal quality to the metrics. CTP [1] is a current treebased routing protocol using information from multiple layers [12]. ODEUR [2] is another promising routing protocol based on detecting the movement of the sensor nodes relative to the data sink. Its disadvantage is that by design, it cannot forward the beacon over more than 2 hops; therefore its scalability in WSNs is limited. A comparison between RSSI (Received Signal Strength Indicator) and LQI (Link Quality Indicator) based on the PRR is discussed in [6]. In that publication, RSSI is discussed as a better indicator of delivery probability than LQI.

The inspiration for our new routing protocol, ODEUR ${ }^{+}$, is motivated by the idea that applying WSNs with a combination of other technologies (RFID, GPS, etc) to logistics can enhance an intelligent transportation system. Every item is uniquely identified, from the warehouse to the containers and at the destination, with the help of WSNs. The objective here is to define a suitable model of WSNs in logistics to provide a means for collecting the information of goods in containers during the transportation. In order to satisfy the requirements of dynamics in logistics, the goals of our routing protocol are:

- Use the RSSI, which is available in many hardware platforms, for routing. RSSI is a good indicator representing the receiving signal quality.

- Make movement estimations of the neighboring sensor nodes and use this information for routing.

- Use as little memory as possible because of the hardware resource limitations in sensor nodes.

- Support multiple sinks so that sensor nodes can automatically connect to available sinks when they move through many WSNs.

- Use a simple mechanism to synchronize the network time.

- Support a backup route in case there are problems related to loops and duplicate packets with the working route.

- Only keep the good neighbors in the neighbor table by classifying the quality of neighbors.

- Use retransmissions and acknowledgements to improve the reliability. 
The contribution of this paper is not only a model of the routing protocol for sensor networks but also an application which can be used in logistic scenarios. To validate the model, a simulation environment for logistics is built and the performance of the proposed protocol is shown to match the design goals.

This paper is structured in 6 sections: section 1 is the introduction to the scope of this paper, section 2 describes the operation of the protocol, and section 3 shows the model of this protocol as well as the functionalities of its components. Formats of beacon and data packets are proposed in section 4. Simulation results are shown in section 5 and finally conclusions are given at the end of this paper.

\section{ODEUR ${ }^{+}$- An enhanced routing protocol based on ODEUR}

$\mathrm{ODEUR}^{+}$is a destination-based routing protocol utilizing the advantages of ODEUR to consider the movement of nodes by using a metric called Mobility Gradient (MG) and RSSI for the routing. The MG metric represents the relative movement between nodes. In order to transmit data packets from sensor nodes to the sink (destination), there are two processes: beacon broadcasting to exchange the routing information in the network and sending of data packets when the routing information is available.

\subsection{Broadcasting beacons}

Our protocol ODEUR ${ }^{+}$uses a periodical beacon to signal the routing information from a sink to the network to build a collection tree. Here, the original ODEUR has a disadvantage which is the 2-hop forwarding problem: beacons only are forwarded over a maximum of 2 hops. As for further hops, the mobility in relation to a sink cannot be determined reliably. So in the original ODEUR, the scalability of the network is limited. In order to overcome this problem, every node in the WSN will perform the multi-hop forwarding mechanism in ODEUR ${ }^{+}$. After receiving the beacon, each node extracts the RSSI and MG (the MG is calculated by subtracting two continuous RSSI values as in [2]) of the beacon sender to update its neighbor table and then sends a new beacon with new information to its neighbor. Therefore, different from ODEUR, each sensor node knows the relative movement of all the neighbor nodes (and 2-hop neighbor nodes), but not necessarily that of a sink. In ODEUR ${ }^{+}$, the sink movement is not taken into account because it is not necessary in a large network where a node should keep the information of the neighbor nodes instead those far away. Unlike ODEUR, ODEUR ${ }^{+}$uses a multi-hop forwarding mechanism, with the power of the sink beacons being the same as the power of data packets. 


\subsection{Sending data}

After building the neighbor table, each sensor node will choose the best neighbor node $(\mathrm{BNN})$ to transmit data packets.

In order to describe the applied routing function, a simple topology is shown in figure 1 with a neighbor table of node 4 after updates. The following procedure will do the BNN selection based on the information provided by the neighbor table:

Fig 1: Opportunistic routing: source RSSI (srcRSSI) and MG (srcMG) are the RSSI and MG values of the beacon sender, which are calculated from received beacons. Neighbor node RSSI (nnRSSI) and MG (nnMG) are available in received beacons through routing information exchange.

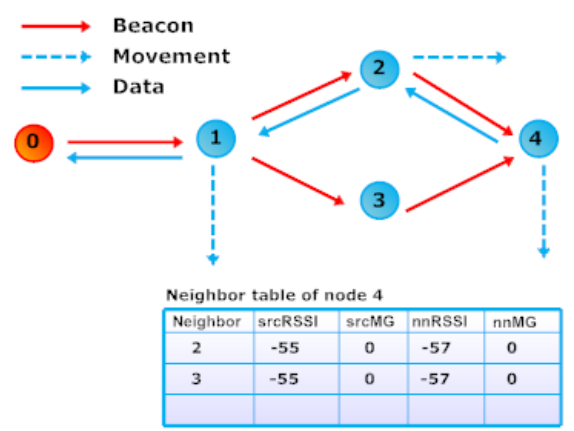

- Normally, if the neighbor node or the source node is in the communication range of the sink, the source node will choose the sink or the neighbor node to communicate directly.

- In a network with multi-hop transmission, the source node (in the example of Fig. 1 node 4) tries to choose the neighbor which has the strongest RSSI to forward packets because this is its closest node. At the beginning, the source node will check the 1-hop neighbor nodes. Assuming that RSSI value of several 1-hop neighbor nodes (node 2 and 3 in the example) is the same, and they move, the node moving towards the source node is selected. In the example node 2 moves towards node 4 , the MG value ( $\operatorname{srcMG}$ ) will be +1 to indicate the direction of movement is "closer". Node 4 will choose node 2 as BNN. Otherwise, the MG is -1 to indicate the direction is "further", node 4 will choose node 3 as BNN because it is better. The same selection is performed if node 4 moves and node 2 , and 3 do not move.

- If there are no changes in 1-hop neighbor nodes ( $r r c M G$ and srcRSSI do not change), the source node will check the nnRSSI and nnMG to choose the BNN. For example, if node 1 moves towards node 3 , the nnMG in the table of node 4 will be +1 for node 3 and -1 for node 2 so node 4 will choose node 3 as BNN although in this case, the RSSI of node 3 and 2 is the same (assuming that node 2, 3 and 4 do not move).

\section{Model}

Based on the previous description, a model of the routing system (shown in 
figure 2) is proposed with many advantages. It has three components: the neighbor management, the routing and forwarding components with separate functions so that the modification of each component does not affect the others. The neighbor management component is responsible for receiving beacons and validating these beacons to avoid loops or duplications. After that, the information in a valid beacon is used to update the neighbor table with a management policy: the insert or update rate is normally equal to the beacon rate, but the evict rate is defined by a timer using the frequency algorithm [7] to count the number of received beacons from each neighbor and use it as a quality indicator. This can help to classify the neighbor nodes: only the high-quality nodes, from which the current node receives many update beacons, are kept in the neighbor table. The low-quality nodes, which send less update beacons, are removed step by step to save the memory for the better nodes.

Fig 2: Model of routing layer

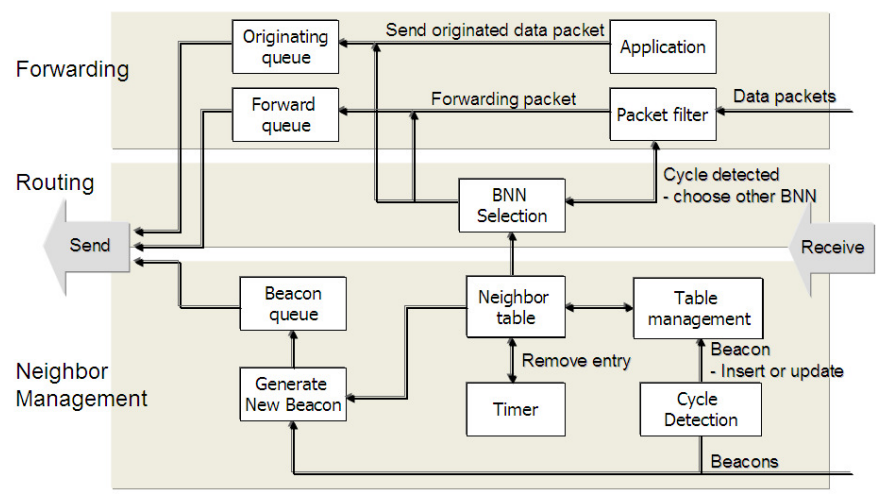

In the routing component, a routing function is implemented to choose the best neighbor node (BNN) based on the information provided by the neighbor table. In addition, in order to have a higher reliability, a backup BNN is also assigned if possible. This component can easily be changed to implement different routing algorithms without affecting other components.

The forwarding component performs two tasks: sending the data packets from the application layer and forwarding the packets coming from the other nodes. It has a packet filter component to validate the forwarded packets do not create loops or are duplicated. If an incoming packet is valid, it is forwarded to a chosen BNN in the routing component, otherwise the current node can decide whether it uses the backup BNN or discards this packet.

Every node in a WSN can also be a forwarding node; therefore the forwarded data traffic volume (from other nodes) is usually higher than the originated data traffic volume. Thus, separated buffers can divide the traffic to avoid the dominance of the forwarding traffic over the originated traffic. Moreover, with the separation of buffers, it is easier to apply a user scheduling mechanism. 


\section{Packet formats}

Two types of packets are used in this protocol: beacons for routing and data packets for collecting information from sensor networks. The following sections show the proposed formats of these and their meanings.

\subsection{Beacon format}

- Sink ID (2 bytes): address of the sink where the beacon originated

- Sender (2 bytes): the address of the node which forwards the beacon

- Sequence (2 bytes): a unique number to identify the beacon

- RSSI ( 2 bytes): RSSI value which the sender measured after receiving the beacon on the previous hop

- $M G$ (1 byte): MG value which the sender determined after receiving the beacon

- Network time (4 bytes): this value is used to synchronize the local timers at sensor nodes. This assumes the delay of PHY and MAC layers can be neglected.

- Neighbor-Num (1 byte): the number of nodes which can communicate directly with the sink.

- Neighbor-List (10 bytes): addresses of the nodes which can directly communicate with the sink. This list can be segmented to fit well in this field.

\subsection{Data packet format}

- Mote ID (2 bytes): the address of sensor node which originates this packet

- Sink ID (2 bytes): address of destination sink

- Time-to-live (TTL) (1 byte): is used to eliminate loop problems in the network.

- Sequence (2 bytes): is used to recognize and avoid duplicated packets.

- Data ( $n$ bytes): any transmitted information

To avoid the problem of loops or duplicate packets, a cross-layer technique is used in this model. Although the fields of the data packet are on the application layer, the routing layer can use this information by cross reading the header information for its use. And these two types of packet fit well in the active message layer [13].

\section{Simulation}

In the simulation, an area with the size $300 \mathrm{~m} \times 300 \mathrm{~m}$ is used to represent a logistical scenario. The positions of nodes in these areas can be fixed or random. Every node can be static or mobile with a given speed between 2 and $5 \mathrm{~m} / \mathrm{s}$. The path loss of the signal in the simulation uses a free-space model with the specifica- 
tions of the CC2420 radio chip. Every node will transmit the monitored temperature to the sink after a specific period. All scenarios are simulated in TOSSIM [8]. Although in TOSSIM, the signal power is a constant [9], a matrix of gain between nodes is developed and can be varied in each step of the simulation. With that improvement, any propagation model can be applied in the simulation.

\subsection{Scenario 1: network with mobility}

In this scenario, a single WSN is used for simulation, which is representing a logistic warehouse where there are many containers needing to be transported by ships. Let us assume that each container is represented by a sensor node and the container can be moved to the warehouse to one location for storage. It can also be moved out for transportation. In order to simulate a more general mobility scenario, the movements of the containers are random paths. All the parameters are measured at the sink during the simulation time of 6 hours.

In order to find the optimum beacon period, some cases are simulated (static

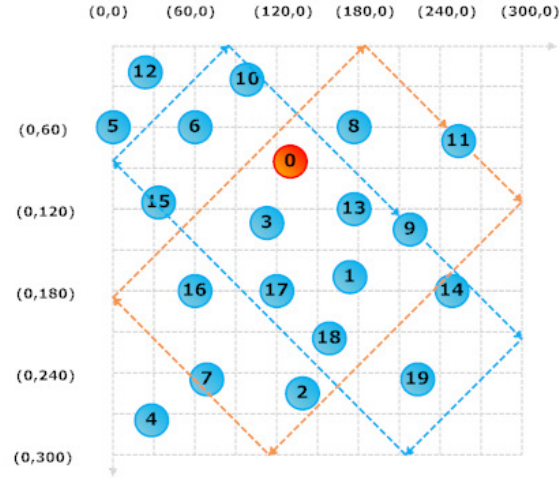

Figure 3: Random layout of nodes in the area $300 \mathrm{~m}$ x $300 \mathrm{~m}$. Every node can move in a random path. When reaching the boundary, it will change the direction following the reflecting law and mobility) in an area $300 \mathrm{~m} \times 300 \mathrm{~m}$ with the data rate of 1 packet/2s (each packet has 29 bytes). It can be seen in figure 4 that the optimum value is in the range of 2-8 seconds to keep the packet reception rate at nearly $90 \%$. If the period is too short, the physical bandwidth is mostly used for transmitting beacons instead of data packets. However, with long beacon periods, the routing will not adapt to the changes of the network.

Fig 4: The optimum beacon period is between 2 and 8 seconds. Any value out of this range gives a poor PRR.

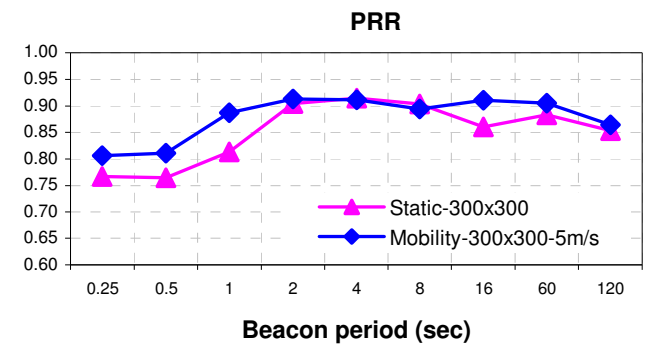

The maximum data rate is shown in figure 5, in which the data period is varied from $0.5 \mathrm{~s}$ to $60 \mathrm{~s}$, and every node is static with a beacon period of $4 \mathrm{~s}$. In order to achieve the PRR of nearly $90 \%$, the data period should be greater than 2 s with a density of 20 nodes in the area $300 \times 300 \mathrm{~m}$. With a density of 30 nodes in this area, 
the maximum PRR is approximate $85 \%$ at the data period of $5 \mathrm{~s}$.

Fig 5: The optimum data period is $2 \mathrm{~s}$ at the density of 20 nodes in an area 300 $\mathrm{m} \times 300 \mathrm{~m}$

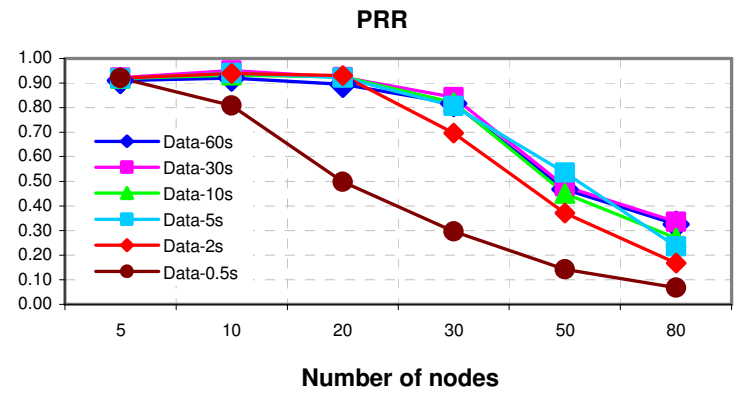

The loss of packets depends on the propagation and on the buffers in each node. In order to measure the buffer loss, the number of incoming packets, outgoing packets and the duplicate packets are monitored at each sensor node. From these, the buffer loss is calculated. Our simulations also show that the buffer loss of every node is rather low (under 1.5\%) because of the separate buffers (beacon, forwarding and originated packets). This means that the loss is mostly because of the coverage of the signal.

Moreover, in our simulations, the memory is also taken into account. The size of the routing table is 10 entries ( 1 entry has only 13 bytes). Hence, this low usage of memory is significant in many hardware platforms which have limited resources.

\subsection{Scenario 2: multiple WSNs in logistic harbor scenario}

In this section, a logistic scenario in a harbor is used for simulation (figure 6). The containers will be moved from the storage house to the crane area, and after that they will be loaded into the ship. 3 WSNs can be used in this scenario, so that every container can connect to transmit the data packets which contain the environment condition information monitored by the sensors inside the container.

Fig 6: Transportation of containers in a harbor

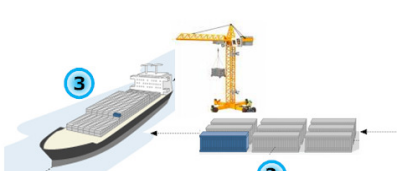

(2)

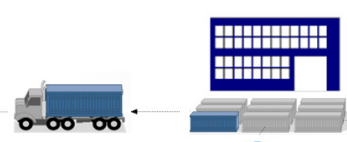

(1)

A mapping scenario shown in figure 7 is created to simulate the communication progress of the moving container. There are 4 areas: 1, 2 and 3 where the moving sensor node (container) can connect to send packets and area 4 is the range in which sensor nodes cannot connect because of a very weak signal. The sink addresses in area 1,2, and 3 are 0,20 and 40. The positions of sensor nodes in these WSNs can be random or deterministic. The mobile node (address 60) has the speed of $2 \mathrm{~m} / \mathrm{s}$ and moves through 3 sensor networks. 

ping scenario - multi WSNs

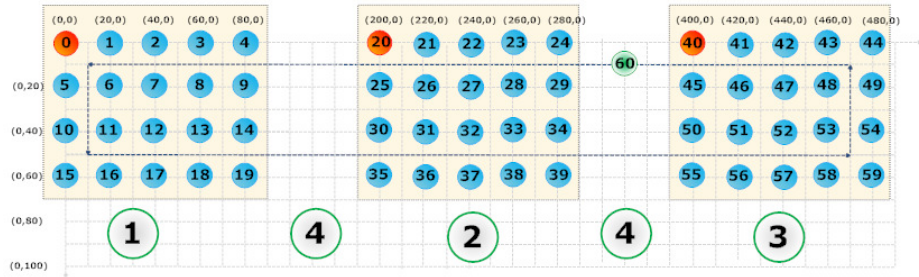

The communication of the mobile node is recorded at each sink to which it connects by counting the number of packets that the appropriate sink receives from sensor node 60 . The result (in figure 8) shows that when the mobile node connects to the sink $(0,20$, or 40$)$, the number of packets received by that sink increases, otherwise (when the mobile node is in other areas) this value is kept constant.

Fig 8: when connected with a sink, the mobile node will transmit packets to this sink, therefore the number of packets received by the appropriate sink increases

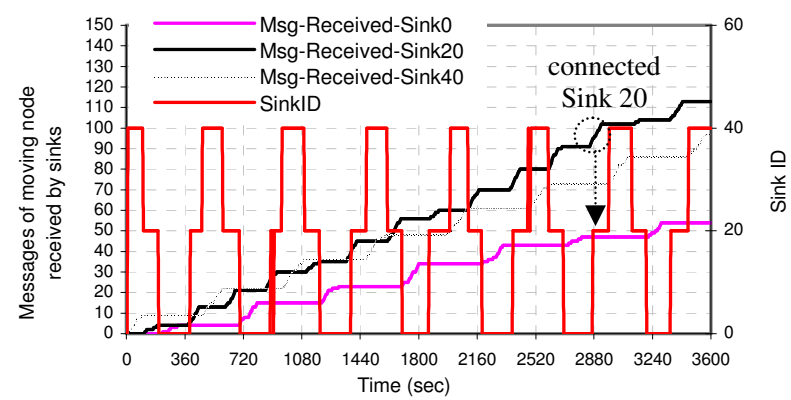

\subsection{Confidence interval}

To validate the reliability of the simulation results, 3 cases are simulated 10 times with 10 different seeds to measure the mean of PRR shown in table 1 to find the confidence interval. The variance is very small and similar in the cases of static networks. With a random network, depending on the random initialization, the variance is bigger than in the other cases.

Table 1: Confidence Interval.

\begin{tabular}{llllll}
\hline Case & Mean & Variance & $\mathrm{Cl} 90 \%$ & $\mathrm{Cl} 95 \%$ & $\mathrm{Cl} 99 \%$ \\
\hline Static-100x100-21nodes & 0.92 & 0.00002302 & $0.92 \pm 0.00278$ & $0.92 \pm 0.00343$ & $0.92 \pm 0.00493$ \\
Static-100x100-21nodes-Mobility-Sink-2m/s & 0.91 & 0.0000191037 & $0.91 \pm 0.00253$ & $0.92 \pm 0.00312$ & $0.92 \pm 0.00449$ \\
Random-300x300-21nodes-Mobility-2m/s & 0.84 & 0.000361535 & $0.84 \pm 0.01102$ & $0.84 \pm 0.0136$ & $0.84 \pm 0.01954$ \\
\hline
\end{tabular}

\section{Conclusion}

In this paper, a flexible model of the routing protocol ODEUR ${ }^{+}$is presented as well as its applications in the logistic harbor scenario. It is believed that this 
model is also well-suited for many applications where mobility is considered.

The simulation results show that the performance of this protocol is rather good, the PRR is nearly $90 \%$ in most cases examined. A set of parameters for configuration is also investigated to achieve the highest PRR. A small scale sensor network (12 nodes) was also deployed physically in our department and reported a significant amount of data with over $95 \%$ packet delivery.

\section{Acknowledgements}

I gratefully acknowledge the fruitful discussions with the other members at TZI ComNets, University of Bremen, Germany. This research is partially funded by the German Research Foundation (DFG) within the Collaborative Research Centre 637 "Autonomous Cooperating Logistic Processes: A Paradigm Shift and its Limitations" (SFB 637) at the University of Bremen, Germany.

\section{References}

1. Collection Tree Protocol - TEP123, www.tinyos.net/tinyos-2.x/doc/html/tep123.html

2. B.-L. Wenning, A. Lukosius, A. Timm-Giel, C. Görg, S. Tomic (2008): Opportunistic Distance-Aware Routing in Multi-Sink Mobile Wireless Sensor Networks. ICT-MobileSummit 2008, Stockholm.

3. A.Timm-Giel, K. Kuladinithi, M. Becker, C. Görg (2006): Wireless Sensor Networks in Wearable and Logistic Application - CRUISE Workshop, page 2, June, 2006, Greece.

4. L. Evers, M. J. J. Bijl, M. Marin-Perianu, R. Marin-Perianu, P. J. M. Havinga (2005): Wireless Sensor Networks and Beyond: A Case Study on Transport and Logistics, page 1, IWWAN 2005, International Workshop on Wireless Ad-hoc Networks, London.

5. Hans-Christian Müller - Wireless Networks in Logistic Systems (2005): Workshop on RFID and Wireless Networks in Logistic systems - November 16th, Duisburg, 2005

6. Kannan Srinivasan, Philip Levis (2006) - RSSI is Under Appreciated. In Proceedings of the Third Workshop on Embedded Networked Sensors (EmNets 2006)

7. E. D. Demaine, A. Lopez-Ortiz, J.I.Munro (2002): Frequency estimation of internet packet streams with limited space. In Proceedings of the 10th Annual European Symposium on Algorithms ESA 2002, pages 348-360, September 2002

8. P. Levis, N. Lee, M. Welsh, D. Culler (2003): TOSSIM: Accurate and Scalable Simulation of Entire TinyOS Applications. In Proceedings of the First ACM Conference on Embedded Networked Sensor Systems (SenSys 2003).

9. T. Rusak, P. Levis (2008): Investigating a Physically-Based Signal Power Model for Robust Wireless Link Simulation. In Proceedings of the 11th ACM International Conference on Modeling, Analysis and Simulation of Wireless and Mobile Systems (MSWiM), 2008.

10.P.Levis, E.Brewer, D.Culler, D.Gay, A.Woo et al (2008): The Emergence of a Networking Primitive in Wireless Sensor Networks. In Communications of the ACM, Volume 51, Issue 7, page-99-106, July 2008.

11. C. Intanagonwiwat, R. Govindan, and D. Estrin (2000): Directed Diffusion: A Scalable and Robust Communication Paradigm for Sensor Networks. Sixth Annual International Conference on Mobile Computing and Network-ing (MobiCom), 2000.

12. R. Fonseca, O. Gnawali, K. Jamieson, P. Levis (2007): Four Bit Wireless Link Estimation, page 1. In Proceedings of the Sixth Workshop on Hot Topics in Networks, 2007.

13. message_t - TEP111, www.tinyos.net/tinyos-2.x/doc/html/tep111.html

14. A.Woo, T. Tong, D. Culler (2003): Taming the Underlying Challenges of Multihop Routing in Sensor Networks. First ACM Conference on Embedded Networked Sensor Systems, 2003. 